\title{
OBSERVATIONS ON THE CAPILLARIES OF THE NORMAL INFANT *
}

\author{
KATHERINE M. MAYER \\ CHICAGO
}

While seeking a method to determine the capillary blood pressure, Lombard ${ }^{1}$ discovered that the circulation in the capillaries of the living person could be observed with a microscope. The skin was rendered transparent with either glycerin or cedar oil. Magnifications of from ten to twenty-five times were found best. For illumination he used either a Nernst lamp or daylight. Later Weiss ${ }^{2}$ observed the capillaries of persons suffering from various diseases. In his method the electric light rays pass through a series of lenses before striking the area of skin to be illuminated; later, he used only one lense. ${ }^{3}$ In determining capillary blood pressure, Danzer and Hooker ${ }^{4}$ used merely a strong electric light and castor oil with their pressure apparatus and objective 3 and ocular 4 of the microscope.

The apparatus used to make my observations is very simple and not bulky. It consists of a small electric lamp (Fig. 1) with a strong convex lense made in the bulb. ${ }^{5}$ The lamp is attached to the objective of the microscope by a metal attachment, in such a way that the light and the microscope can be focused on the same area. Objective 3 and ocular 2 were found best. The area to be observed is first cleaned with green soap, then with alcohol, and well dried before the glycerin or cedar oil is applied. The oil renders the skin transparent by penetrating the epidermis and so doing away with the light reflections from the uneven surface. In very young children, the oil can be applied directly without the preliminary cleaning. The third finger of the infant is strapped loosely, with a narrow strip of adhesive plaster to a wooden tongue depressor. With slight pressure on the knuckle the finger can be held quite firmly without interfering much with the circulation at the nail fold. Observations are made in the area about the middle of the nail fold. The infants were taken after the morning feeding, when they

* Received for publication, June 30, 1921.

* From the Otho S. A. Sprague Memorial Institute Laboratory of the Children's Memorial Hospital.

* Read before the Chicago Pediatric Society, March 22, 1921.

1. Lombard, W. T.: Am. J. Physiol. 29:335, 1912.

2. Weiss, E.: Deutsch. Arch. f. klin. Med. 119:1, 1916.

3. Weiss, E.: Ztschr. f. Exper. Pathol. u. Therap. 19:390, 1918.

4. Danzer, C. S., and Hooker, D. R.: Am. J. Physiol. 52:136, 1920.

5. This lens was furnished by American Specialty Co. 
would most likely sleep or be quiet. Needless to say, it is impossible to make observations when the child is irritable or very restless.

In 1892, Spalteholz ${ }^{6}$ studied the distribution of the blood vessels in the skin by injecting the vessels with a colored gelatinous solution. Among his conclusions is the following statement:

All the vessels which go to the papillae originate from the anastomizing vessels in the subcapillary plexus, and always in the form of small trunks, whose smallest branches run a short distance in the direction of the papillary rows, perpendicular under these, and run without further anastomoses and send their capillaries into the papillae. . . . (This coincides with my observations.) All arteries leading to the skin and their larger branches are the same in distribution and number in the new-born.

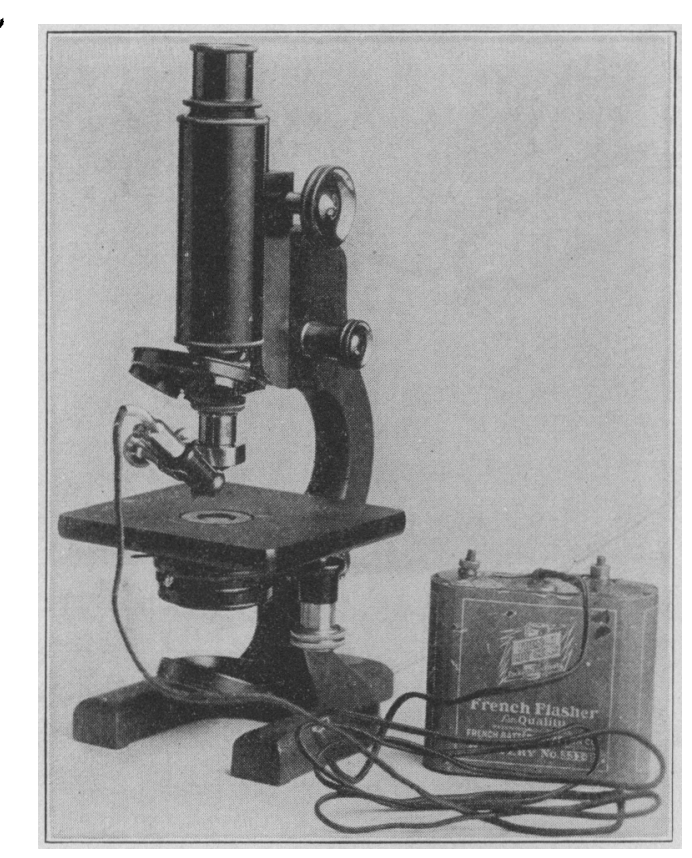

Fig. 1.-Microscope with lamp attached.

He makes no statement concerning the end capillaries of the skin in the new-born.

In 1919, Holland and Meyer ${ }^{7}$ observed the capillaries in healthy normal children, and in those with exudative diathesis. They used the method described by Weiss. They describe and illustrate their findings in detail as follows:

6. Spalteholz, W.: His Arch. f. Anat. u. Entwickelungsgesch. 1: 1893.

7. Holland, M., and Meyer, L.: München. med. Wchnschr. 66:1191, 1919. 
In two-day old infants the end loops do not extend into the limbus (tissue between subcapillary plexus and skin surface), but remain in the region of the subcapillary plexus and extend up from there as short loops in the process of development. Arterial and venous limbs are clearly differentiated. Occasionally one finds a figure of eight loop. At one week regularity in distribution is seen. The short loops do not extend into the limbus. The venous limb is absolutely not differentiated from the arterial limb. (This differs from their findings at two days.) At four weeks the capillaries are of various widths, some are close together and with rich anastomosis, and some are more widely separated. Pronounced differences in length do not exist. The vessels project into the limbus.

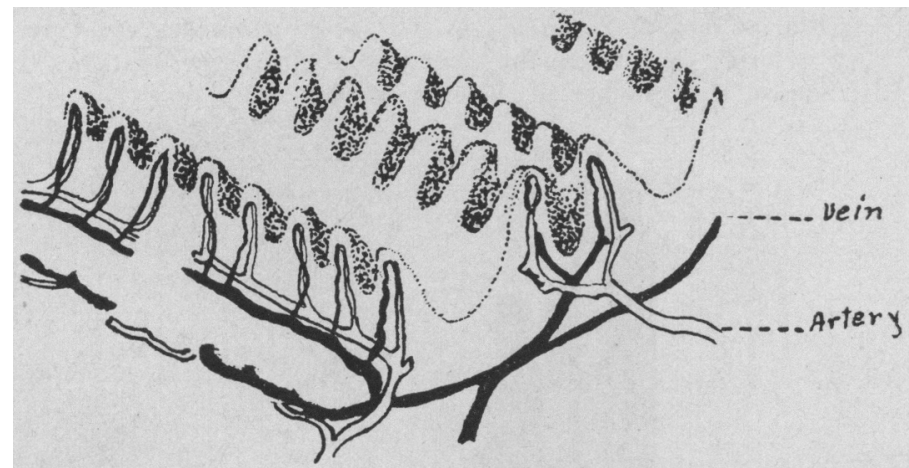

Fig. 2.-The uppermost part of the pars papillaris cutis without the epidermis (Spalteholz).

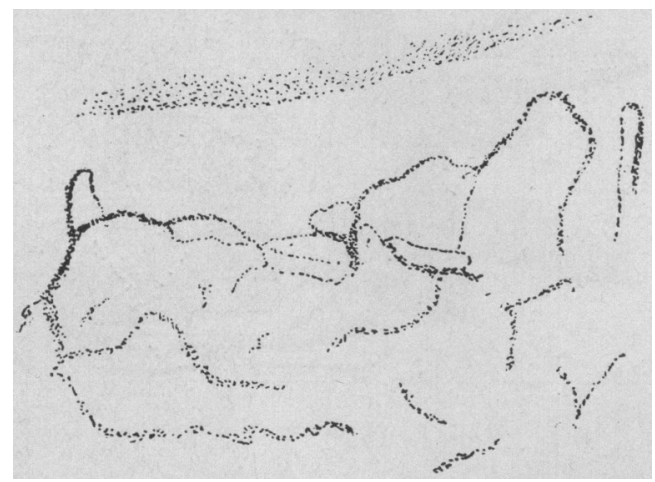

Fig. 3.-Normal infant, 2 days old.

They describe the picture in the normal young infant as follows:

The width of the capillaries is from 0.03 to $0.05 \mathrm{~mm}$. and length $0.15 \mathrm{~mm}$. Difference in length and variation in width is seldom found, and then the difference is not marked. Well marked serpentine forms are seldom found, but, as a whole, there is a tendency to greater irregularity and serpentine forms. The vessels run straight, but not exactly parallel. Markedly twisted loops are seldom found. The arterial and venous limbs are almost always 
well differentiated. In almost all children, the subcapillary plexus is clearly seen. Anastomosis one seldom finds among the end capillaries, but the subcapillary plexus is rich in anastomosis.

Later, Mertz ${ }^{8}$ published his observations. He divides his results into three groups; first, comprising the children from 1 to 3 months of age; second, from 4 to 6 months, and third, those over 6 months.

In the first four or six weeks the loops are very short and project up from the ground substance into the limbus, more as broad bow shaped protrusions than as short loops. It is only the distal part of these loops that one can term the capillary loops. After several weeks there is found a better develop-

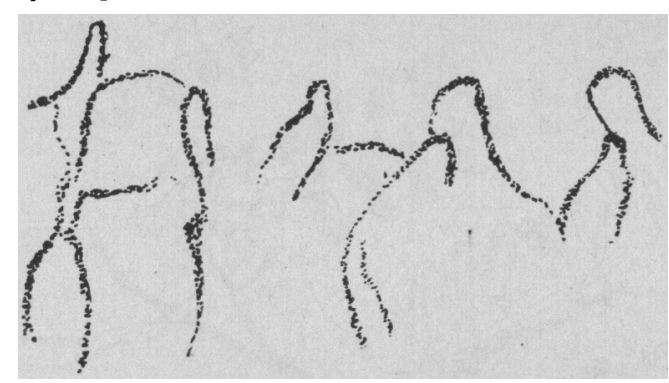

Fig. 4.-Normal infant, 7 days old.

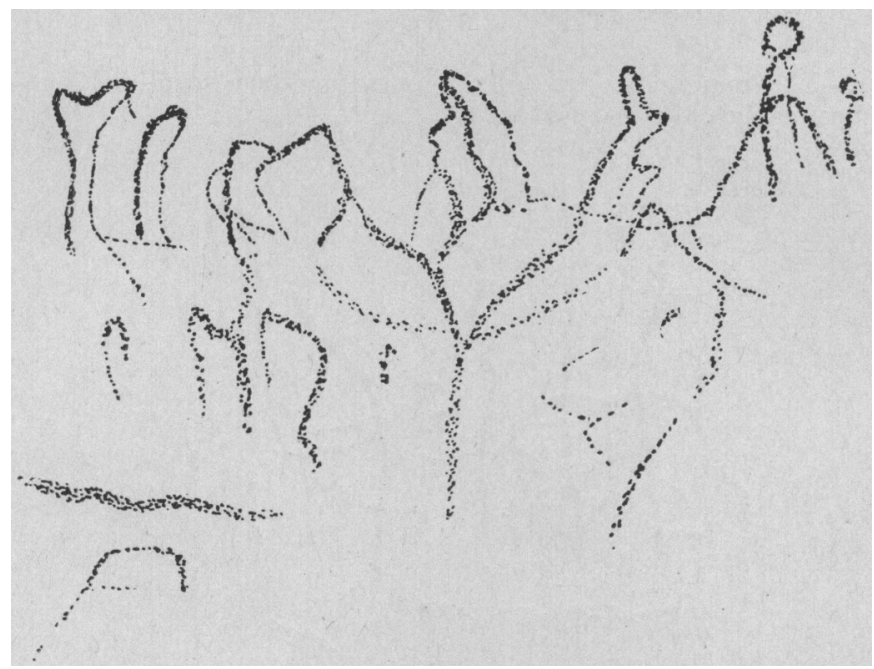

Fig. 5.-Normal infant, 3 weeks old.

ment of the capillaries and an extension into the skin along the edge of the nail fold. The age when this definite development occurs seems dependent on the development of the child. In the first three months, the capillary loops are from 20 to 60 microns long, are relatively not close together and anastomosis is seldom seen. In the following period, from 3 to 6 months, there is a rapid lengthening of the loops; they reach 200 microns. The loops are closer together and have greater anastomosis than before.

8. Mertz, A.: Monatschr. f. Kinderh. 18:13, 1920. 
He describes a variation in length of the capillaries of children of the same age and in the same child on different fingers, this being related to the length of the nail fold; if it is pushed back, the loops are shorter than if it is grown further out over the nail.

My observations were made on infants at the Children's Memorial Hospital and at St. Vincent's Orphan Asylum. Only those infants were examined who appeared to be in good condition. No child with

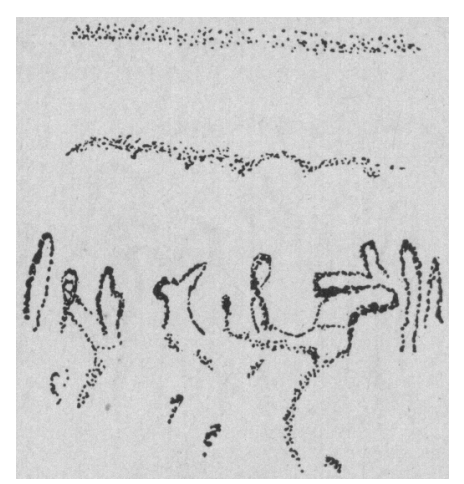

Fig. 6.-Normal infant, 5 weeks old.

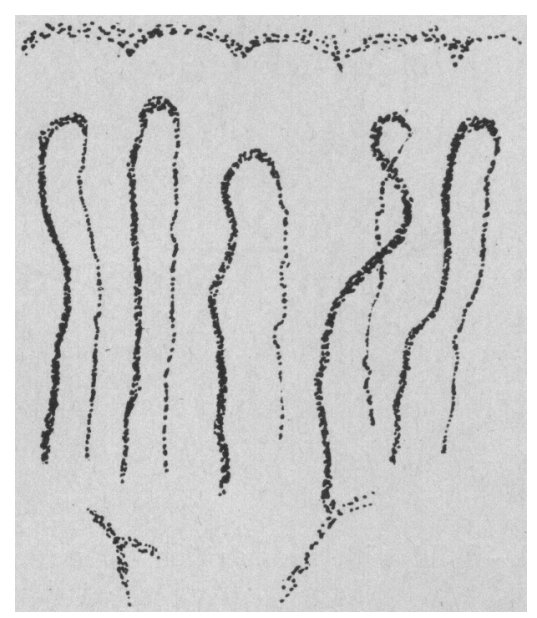

Fig. 7.-Normal infant, 9 months old.

an infection, however slight, with eczema, or in poor physical development was taken as a normal child. Many infants could not be observed as they became too irritable when an attempt was made to hold the hand absolutely quiet under the objective of the microscope. It was found most satisfactory to hold the infant in a sitting position in such a way that the hand could rest comfortably on the stage of the micro- 
scope. Often the infant slept quietly during an entire observation. Drawings were made of those infants who held absolutely quiet for a relatively long time.

In infants 1 or 2 days old (Fig. 3 ) the capillaries appear as a fine network plexus lying close to the skin surface. This plexus can be seen extending back from the edge of the nail fold for quite a distance. Only the most distal vessels of this plexus can be called end capillaries. These are bow shaped, vary in length and height, and the arterial and the venous limbs can be differentiated. The arterial limb is hairlike

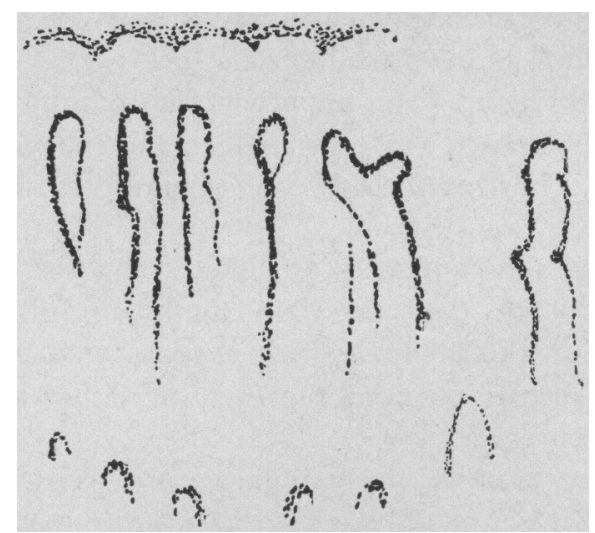

Fig. 8.-Normal adult.

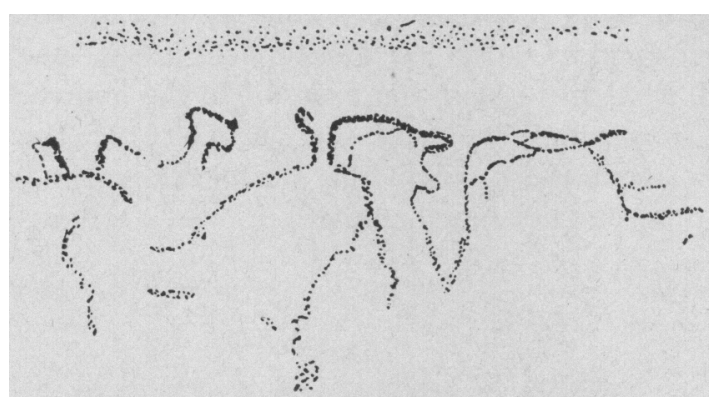

Fig. 9.-Child with malnutrition, 10 weeks old.

and broadens gradually into the venous limb. There is no regularity in the distribution of these loops along the plexus margin. Occasionally, there is a short narrow loop. There is no anastomosis except in the subcapillary plexus.

At one week (Fig. 4) the capillaries begin to elongate and project out into the tissue between the plexus and the skin surface. In some the loops appear triangular, the angle appearing at the top and the base remaining broad. Other loops may be somewhat square. The 
capillaries begin to appear more loop than bow shaped and they are slightly more regular in their distribution. There is no anastomosis between these and loops. When such seemed to be present it was found upon focusing that one loop was superimposed on another. The arterial and venous limbs are clearly differentiated.

At three weeks (Fig. 5) the loops are definitely formed and are separate from each other at relatively equal distances, one loop extending into each papilla. The loops vary in length and breadth, many loops being still quite broad and short. No anastomosis occurs except in the subcapillary plexus, that is, the vessels at the base of the loops. The arterial limb of the capillary vessel is hairlike and broadens out into the venous limb before turning back on itself. The venous limb often is slightly tortuous. The apparent anastomosis in Figure 6 was found to be superimposed vessels in which the two limbs were not in the same plane. There is little if any variation in the caliber of the different vessels.

At two months the capillaries are longer and the distance between the subcapillary plexus and the skin surface is also greater. The vessels disappear in the plexus, which appears as a pinkish haze with here and there a deeper red line, representing either a larger vessel or one closer to the surface.

At about three months, the capillaries are well formed; they are longer than those at two months. There are some spiral and figure of 8 shaped vessels. As the capillaries increase in length, they appear much closer together. From three months on the vessels increase in length until the long narrow loops of the adult type (Fig. 8) are reached. The subcapillary plexus shows less anastomosis at the base of the loops.

In general, it might be said that from birth the end capillaries begin development from small loops at the periphery of the subcapillary plexus. Until about the third month the development is very rapid. From then on there is not much change.

I wish to thank Dr. Samuel Amberg for his direction in this work. 